\title{
Comparison of Dominant and Non-dominant Range of Motion in Collegiate Baseball Pitchers: A Pilot Study
}

\author{
K. Jason Crandall*, Taylor Carlin, Abby Flynn \\ School of Kinesiology, Recreation, and Sport, Western Kentucky University, United States
}

Copyright $(2015$ by authors, all rights reserved. Authors agree that this article remains permanently open access under the terms of the Creative Commons Attribution License 4.0 International License

\begin{abstract}
Shoulder injuries may be prominent in baseball pitchers due to inflexibility of the shoulder and trunk. Differences may exist between pitchers' dominant and non-dominant shoulders and trunk. The purpose of this pilot study was to confirm prior research to compare dominant and non-dominant shoulder and trunk range of motion in fifteen ( $\mathrm{n}=15,18-23$ yrs.) collegiate pitchers. Trunk range of motion was measured using the Y-Balance Test. A goniometer was used to assess seven measurements of shoulder range of motion. Paired sample t-tests were used to determine statistical significance $(p<.05)$. Shoulder extension $(\mathrm{t}(14)=-3.02, \mathrm{p}=.009)$, internal rotation $(\mathrm{t}(14)$ $-2.8, \mathrm{p}=.013)$, horizontal abduction $(\mathrm{t}(14)=-2.17, \mathrm{p}=.047)$, and Y-Balance Test $(\mathrm{t}(14)=-2.2, \mathrm{p}=.044)$ were significantly greater in the non-dominant arm. The findings of our study suggest collegiate baseball coaches and trainers should create strength and conditioning programs that are balanced and focus on both dominant and non-dominant shoulder and trunk range of motion.
\end{abstract}

Keywords Range of Motion, Baseball, Training, Injuries, Pitchers

\section{Introduction}

A successful baseball pitch is achieved through the perfect balance of joint mobility, muscular stabilization, and extreme force [1]. Because the overhead pitching motion places significant physical stress on the dominant shoulder joint, elbow joint, and the surrounding muscles even small imbalances can result in acute and chronic injuries. Loss of flexibility and range of motion (ROM) throughout the upper body may lead to injuries. Specifically, participants' dominant arm is subjected to more constant stress during the pitching motion compared to the non-dominant arm. Many athletes can continue to perform at high levels despite significant inflexibility and loss of ROM, yet many become injured or suffer pain throughout their career. For example, $50 \%$ of athletes $9-14$ years of age experience shoulder pain [2]. It is essential that coaches and trainers focus on injury prevention particularly in their dominant arm, to avoid a decreased level of flexibility, but also to prevent the likelihood of injury.

Because the pitching motion requires the entire superior region of the body, it is important to understand how an athlete's trunk rotation is related to ROM in the upper extremities. Although a large percentage of injuries occur in the upper extremities, $12 \%$ of injuries occur in the spine and trunk region [3]. Tightness in the trunk region can inhibit ROM in both the dominant and non-dominant arm. Researchers have concluded that there is generally greater ROM and flexibility in the dominant throwing arm of baseball players versus their non-dominant arm [3]. A combination of inflexibility in the trunk region and upper extremities may decrease the ROM in the dominant arm of pitchers.

The purpose of this pilot study was to confirm prior research findings in this area by comparing dominant/non-dominant shoulder and trunk ROM in collegiate baseball pitchers to help reduce the likelihood of injury or help rehabilitate from past injury. The findings may help baseball coaches and trainers to create strength and conditioning programs that are balanced and focus on both dominant and non-dominant ROM.

\section{Materials and Methods}

\subsection{Participants}

Male volunteer participants $(\mathrm{N}=15, \mathrm{M}=20.13$ yrs., $\mathrm{sd}=$ 1.4 yrs.) from the Western Kentucky University (WKU) baseball team were recruited for this study during the spring 2015 baseball season. Participants included both left-handed and right-handed pitchers. All position players, including those who also pitched, were excluded from the study. Previously injured pitchers were included unless currently undergoing rehabilitation. All participants completed an informed consent and questionnaire to assess demographic information including dominant arm, past or present injuries, and to confirm the player was exclusively a pitcher. The study protocol was approved by the WKU Institutional Review Board (IRB\# 663956-2). 


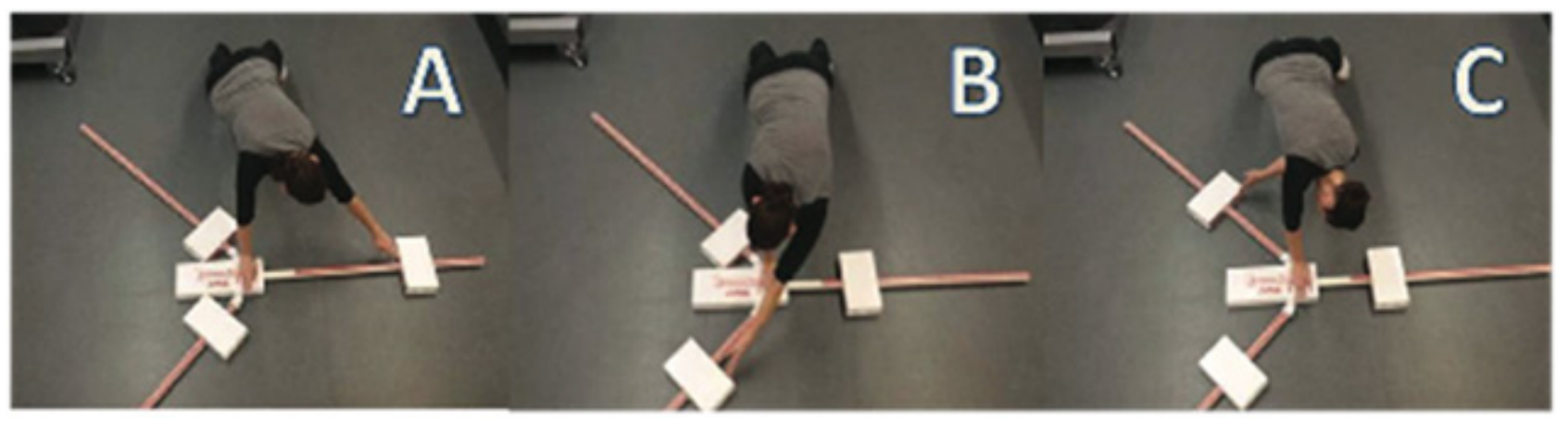

Figure 1. Y Balance Test

\subsection{Outcome Measures}

Testing was completed by the primary investigators during two separate sessions with a minimum of one day between each session. During the first session, participants reported to the WKU Athletic Training room. Participants completed the informed consent and questionnaire. Participants were asked to sit in a chair with good posture and both feet planted on the floor. Shoulder ROM was then assessed using a goniometer. Although some suggest the goniometer should not be used in research settings, it is the most universal and preferred instrument in determining a person's ROM and has been found to be highly reliable [4]. The goniometer was found to be valid when the tester used their anatomical knowledge and visual skills in conjunction with proper technique [5]. Seven measurements were completed: 1) shoulder flexion; 2) shoulder extension; 3) shoulder abduction; 4) shoulder internal rotation; 5) shoulder external rotation; 6) shoulder horizontal abduction; and, 7) shoulder horizontal adduction.

During the second session, trunk ROM was assessed using a Y balance test kit [6]. This test is commonly used as a reliable measure of upper quarter extremity strength, mobility and flexibility. The $\mathrm{Y}$ balance test can also measure a person's risk for injury and their functional symmetry.

Upper limb length was initially measured while the shoulder was abducted to 90 degrees, and elbow was extended fully. The upper quarter Y-balance test, has a series of six dynamic tests to assess shoulder range of motion and isometric trunk rotation. The participant maintained a pushup position and performed maximal reach efforts with each hand, in three separate directions including medial (A), superolateral (B), and inferolateral (C). See Figure 1. Each participant was allowed three practice attempts before the completing the test [6].

\subsection{Statistical Analysis}

Paired sample t-tests were used to compare dominant and non-dominant shoulder and trunk ROM. Statistical significance was set at the .05 level. The statistical software Statistical Package for the Social Sciences (SPSS, version 21.0, Chicago, IL) was used for statistical analyses.

\section{Results}

The purpose of this pilot study was to confirm prior research findings in this area by comparing dominant/non-dominant shoulder and trunk ROM in collegiate baseball pitchers. Shoulder extension $(\mathrm{t}(14)=$ $-3.02, \mathrm{p}=.009)$, internal rotation $(\mathrm{t}(14)=-2.8, \mathrm{p}=.013)$, horizontal abduction ( $\mathrm{t}(14)=-2.17, \mathrm{p}=.047)$, and the Y-Balance Test $(\mathrm{t}(14)=-2.2, \mathrm{p}=.044)$ were significantly greater in the non-dominant arm. Shoulder flexion $(\mathrm{t}(14)=$ $0.39, \mathrm{p}=.700)$, abduction ( $\mathrm{t}(14)=0.56, \mathrm{p}=.586)$, external rotation $(\mathrm{t}(14)=0.88, \mathrm{p}=.394)$, and horizontal adduction $(\mathrm{t}$ $(14)=0.60, p=.561)$ were not significant. See Table 1 for results.

Table 1. Results of Paired Sample T-Test

\begin{tabular}{|c|c|c|c|c|}
\hline Pair & $M$ & $\mathrm{t}$ & $\mathrm{df}$ & $\mathrm{p}$ value \\
\hline Flexion & $-0.67(6.58)$ & -0.39 & 14 & .700 \\
\hline Extension & $-7.24(9.32)$ & -3.02 & 14 & $.009^{*}$ \\
\hline Abduction & $-1.47(10.19)$ & -0.56 & 14 & .586 \\
\hline Internal Rotation & $-8.20(11.19)$ & -2.84 & 14 & $.013^{*}$ \\
\hline External Rotation & $6.47(28.45)$ & 0.88 & 14 & .394 \\
\hline Horizontal Abduction & $-8.40(14.96)$ & -2.18 & 14 & $.047^{*}$ \\
\hline Horizontal Adduction & $-1.53(9.97)$ & -0.60 & 14 & .561 \\
\hline Trunk Range of Motion & $-2.45(4.28)$ & -2.21 & 14 & $.044 *$ \\
\hline
\end{tabular}

Note. ${ }^{*} \mathrm{p}<.05$.

\section{Discussion}

The results of this pilot study confirm others research that found non-dominant shoulder ROM was significantly greater compared to the dominant shoulder in four of the eight measurements. This imbalance in ROM is most likely the result of the aggressive nature of overhand baseball pitching. The reoccurring strain of overhand pitching on the dominant shoulder results in the shoulder muscles becoming increasingly inflexible, thus reducing ROM [1]. Despite the non-dominant shoulder exhibiting an increased overall ROM, external rotation was greater in the dominant 
side since it is a prominent phase during a baseball pitch. The shoulder externally rotates during the pitching motion leading to the increased flexibility seen in external rotation in the dominant shoulder [3].

Not only are the shoulders affected, but research suggests the engagement of the core musculature during a baseball pitch decreases the ROM on the dominant side [7]. The participants exhibited less ROM in their non-dominant trunk side in comparison to the dominant side. This is likely the result of the pitching technique. When pitching a baseball, the entire body is required to move swiftly to exert as much force as possible, therefore the entire core is engaged and twists with the pitch. This twisting motion therefore increased the level of flexibility on the dominant side due to its regular use. These results suggest that pitchers should focus on maintaining an equal level of range of motion in both shoulders and their core to avoid injury [3].

\section{Conclusions}

To help reduce the likelihood of injury or help rehabilitate from past injury, the findings of our study suggest baseball coaches and trainers should create strength and conditioning pro-grams that are balanced and focus on both dominant and non-dominant ROM.

\section{Acknowledgements}

The authors wish to acknowledge Dr. Larry Lowing for his editing assistance. Thomas Peter and Leiff Clarkson were instrumental during the data collection phase of this project.

\section{REFERENCES}

[1] J.M. Downar, E.L. Sauers. Clinical Measures of Shoulder Mobility in the Professional Baseball Player. Journal of Athletic Training, Vol. 40, No.1, 23-29, 2008.

[2] W.J. Hurd, K.R.Kaufman. Glenohumeral Rotational Motion and Strength and Baseball Pitching Biomechanics. Journal of Athletic Training, Vol 47, No. 3, 247-256, 2008.

[3] K. Launder, R. Lynall, J. Williams, R. Wong, T. Onuki, \& K. Meister. Thoracolumbar Range of Motion in Baseball Pitchers and Position Players. International Journal of Sports Physical Therapy, Vol. 8, No. 6, 777-783, 2013.

[4] D. Riddle, J. Rothstein, \& R. Lamb. Goniometric Reliability in a Clinical Setting: Shoulder Measurements. Journal of the American Physical Therapy Association, Vol. 67, 668-673, 1987.

[5] R. Gajdosik, R. Bohannon. Clinical Measurement of Range of Motion: Review of Goniometry Emphasizing Reliability and Validity. Journal of the American Physical Therapy Association, Vol. 67, 1867-1872, 1987.

[6] R. B. Westrick, J.M. Miller, S.D. Carrow, J. P. Gerber. Exploration of the Y-Balance Test for the Assessment of Upper Quarter Closed Kinetic Chain Performance. The International Journal of Sports Physical Therapy Vol. 7, No. 2 139-147, 2012.

[7] P. Anloague, V. Spees, J. Smith, M. Herbenick, J. Rubino. Glenohumeral Range of Motion and Lower Extremity Flexibility in Collegiate-Level Baseball Players. Sports Health: A Multidisciplinary Approach, Vol. 4, No. 1, 25-30, 2011. 